VOL. 63 (2001) [475-484]

\title{
ISOMETRIES OF SPACES OF UNBOUNDED CONTINUOUS FUNCTIONS
}

\author{
Jesús ARAujo and KRZYSZTOF JaRosz
}

\begin{abstract}
By the classical Banach-Stone Theorem any surjective isometry between Banach spaces of bounded continuous functions defined on compact sets is given by a homeomorphism of the domains. We prove that the same description applies to isometries of metric spaces of unbounded continuous functions defined on non compact topological spaces.
\end{abstract}

Let $X, X^{\prime}$ be topological spaces and let $A(X), A\left(X^{\prime}\right)$ be metric vector spaces of continuous functions on $X$ and $X^{\prime}$, respectively. If $A(X), A\left(X^{\prime}\right)$ consist of only bounded functions, for example if $X, X^{\prime}$ are compact, then, in most cases, we have a complete description of all surjective linear isometries $T: A(X) \rightarrow A\left(X^{\prime}\right)$. One may check $[2,3,4,5,6,7,10,11,12,13]$ for descriptions of isometries of spaces of bounded continuous scalar and vector valued functions, bounded analytic functions, absolutely continuous functions, bounded Lipschitz functions, differentiable functions, and many other classes of bounded functions. Usually (but not always, see for example [1] or [9]) any such an isometry is given by a homeomorphism $\varphi: X^{\prime} \rightarrow X$ followed by a multiplication by a continuous scalar valued function $\kappa$; that is,

$$
T f=\kappa f \circ \varphi \text { for all } f \in A(X)
$$

Any map of the above form will be called canonical. The most classical result in this area is the Banach-Stone Theorem which states that any surjective isometry of the space of all bounded continuous functions on a compact set $X$ equipped with the usual sup norm, onto the space of bounded continuous functions on a compact set $X^{\prime}$, is canonical. In this paper we discuss isometries of spaces of unbounded continuous functions.

Assume that $X$ is a $\sigma$-compact topological space and let $X_{1}, X_{2}, \ldots$ be a sequence of compact subsets of $X$ such that

$$
X_{1} \varsubsetneqq X_{2} \subsetneq \ldots \varsubsetneqq X_{n} \subsetneq \ldots, \text { and } \bigcup_{n=1}^{\infty} X_{n}=X .
$$

Received 24th July, 2000

Research partially supported by the Spanish Dirección General de Investigación Cientifica y Técnica (DGICYT, PB95-0582).

Copyright Clearance Centre, Inc. Serial-fee code: 0004-9727/01 \$A2.00+0.00. 
The two metrics most often considered on the space of unbounded continuous functions on $X$ are:

$$
\begin{gathered}
\|f\|=\sum_{n=1}^{\infty} a_{n}\|f\|_{n}, \text { and } \\
d(f, g)=\sum_{n=1}^{\infty} a_{n} \frac{\|f-g\|_{n}}{1+\|f-g\|_{n}},
\end{gathered}
$$

where $\|\cdot\|_{n}$ is the usual sup norm on the set $X_{n}$ and $\left(a_{n}\right)_{n=1}^{\infty}$ is a fixed sequence of positive numbers such that $\sum_{n=1}^{\infty} a_{n}<\infty$; for example, $a_{n}=1 / 2^{n}$. The formula (1) defines a Banach space norm; for fixed sequences $\left(X_{n}\right)$ and $\left(a_{n}\right)$ we shall denote this space by $\widetilde{C}(X)$. The space $\widetilde{C}(X)$ contains unbounded continuous functions of restricted rate of growth; it does not contain all the continuous functions. The second formula defines a complete metric on the space $C(X)$ of all the continuous functions on $X$. In this paper we prove that all surjective isometries of the spaces $(\widetilde{C}(X),\|\cdot\|)$ and $(C(X), d(\cdot, \cdot))$ are canonical. The results are valid both in the real and in the complex case; however at some crucial points the arguments will be different in the two cases. We denote by $\mathbb{K}$ the set of scalars ( $\mathbb{R}$ or $\mathbb{C})$, and by $\mathbb{K}_{1}$ the set of scalars of absolute value one.

THEOREM 1. Let $(\widetilde{C}(X),\|\cdot\|)$ be the Banach space of continuous functions on a $\sigma$-compact set $X$ with the norm given by (1). Let $\left(\widetilde{C}\left(X^{\prime}\right),\|\cdot\|\right)$ be the analogous space on $X^{\prime}$. Assume that $T$ is a linear isometry from $\widetilde{C}(X)$ onto $\widetilde{C}\left(X^{\prime}\right)$. Then there is a homeomorphism $\varphi$ of $X^{\prime}$ onto $X$ with $\varphi\left(X_{n}^{\prime}\right)=X_{n}$, for $n \in \mathbb{N}$, and a unimodular, continuous, scalar valued function $\kappa$ on $X^{\prime}$ such that

$$
T f=c \cdot \kappa \cdot f \circ \varphi \text { for all } f \in \tilde{C}(X),
$$

where $c=\left(\sum_{n=1}^{\infty} a_{n}\right) /\left(\sum_{n=1}^{\infty} a_{n}^{\prime}\right)$. Moreover, $a_{n}=c a_{n}^{\prime}$, for $n \in \mathbb{N}$.

\section{PROOF OF THE THEOREM}

DEFinition 1: We call a nonempty subset $\Omega$ of $\widetilde{C}(X)$ a peaking set if

$$
\left\|f_{1}+\ldots+f_{p}\right\|=\left\|f_{1}\right\|+\ldots+\left\|f_{p}\right\| \text { for all } f_{1}, \ldots, f_{p} \in \Omega .
$$

A subset of $\widetilde{C}(X)$ is called a maximal peaking set if it is a peaking set and it is not contained properly in any other peaking set.

The definition of a peaking set involves only the norm and the addition operation of the vector space. Since surjective isometries preserve both the metric and the linear structure they map peaking sets onto peaking sets and maximal peaking sets onto maximal peaking sets. 
Definition 2: For a given peaking set $\Omega$ we define the complement $\Omega^{\perp}$ of $\Omega$ by

$$
\Omega^{\perp}=\left\{g \in \widetilde{C}(X): \exists r>0 \quad \exists f \in \Omega \quad \forall \alpha \in \mathbb{K}_{1} \quad\|f+r \alpha g\|=\|f\|\right\} .
$$

Again, linear isometries map complements of peaking sets onto complements of peaking sets.

Definition 3: For $x \in X$ we define the index of $x$ by

$$
\text { ind }(x)=\min \left\{n: x \in X_{n}\right\} \text {. }
$$

We shall say that $\left(x_{n}\right)_{n=1}^{\infty}$ is an increasing sequence in $X$ if ind $\left(x_{1}\right)=1$, and for any $n \in \mathbf{N}$, we have

$$
\text { ind }\left(x_{n+1}\right)>\text { ind }\left(x_{n}\right) \quad \text { or } \quad x_{n+1}=x_{n},
$$

and the set $\left\{x_{n}: n \in \mathbb{N}\right\}$ has no limit points.

LEMma 2. A subset $\Omega$ of $\widetilde{C}(X)$ is a maximal peaking set if and only if there is an increasing sequence $\left(x_{n}\right)_{n=1}^{\infty}$ in $X$ and a sequence $\left(\theta_{n}\right)_{n=1}^{\infty}$ of scalars of absolute value one such that

$$
\Omega=\Omega\left(\left(x_{n}\right),\left(\theta_{n}\right)\right) \stackrel{d f}{=}\left\{f \in \widetilde{C}(X): \forall n \in \mathbb{N},\|f\|_{n}=\theta_{n} f\left(x_{n}\right)\right\} .
$$

Proof: Assume $\Omega=\Omega\left(\left(x_{n}\right),\left(\theta_{n}\right)\right)$. For any $f_{1}, \ldots, f_{p} \in \Omega$, we have

$$
\begin{aligned}
\left\|f_{1}+\ldots+f_{p}\right\| & =\sum_{n=1}^{\infty} a_{n}\left\|f_{1}+\ldots+f_{p}\right\|_{n} \\
& =\sum_{n=1}^{\infty} a_{n} \theta_{n}\left(f_{1}\left(x_{n}\right)+\ldots+f_{p}\left(x_{n}\right)\right)=\left\|f_{1}\right\|+\ldots+\left\|f_{p}\right\|,
\end{aligned}
$$

so $\Omega$ is a peaking set. Assume that $\Omega_{0}$ is a strictly larger peaking set and let $f_{0} \in \Omega_{0}-\Omega$. Since $f_{0} \notin \Omega$ then we have two cases:

1. There is a positive integer $k$ such that $\left\|f_{0}\right\|_{k}>\left|f_{0}\left(x_{k}\right)\right|$, or

2. there is a positive integer $k$ such that $\left\|f_{0}\right\|_{k}=\left|f_{0}\left(x_{k}\right)\right|$, but $\left\|f_{0}\right\|_{k} \neq \theta_{k} f_{0}\left(x_{k}\right)$.

Let $\left(y_{n}\right)$ be a sequence of nonzero scalars such that for any $n,\left|y_{n}\right|=\theta_{n} y_{n}$, the series $\sum_{n=1}^{\infty} a_{n}\left|y_{n}\right|$ is convergent, and $y_{n}=y_{n+1}$ if $x_{n}=x_{n+1}$ and $\left|y_{n}\right|<\left|y_{n+1}\right|$, if $x_{n} \neq x_{n+1}$. In the first case we introduce a continuous function $f$ on $X$ such that

$$
\begin{aligned}
& f(x)=0 \text { for any } x \in X_{k} \text { with }\left|f_{0}(x)\right|=\left\|f_{0}\right\|_{k}, \text { and } \\
& \|f\|_{n}=\theta_{n} f\left(x_{n}\right)=\theta_{n} y_{n}, \text { for any } n \in \mathbb{N} .
\end{aligned}
$$


To construct such a function $f$ we first construct inductively a continuous function $\tilde{f}$ on $X$ : after it is defined on $\bigcup_{j=1}^{n} X_{j}$ we extend it to $\bigcup_{j=1}^{n} X_{j} \cup\left\{x_{n+1}\right\}$ by putting $\widetilde{f}\left(x_{n+1}\right)=y_{n+1}$, and then extend to $\bigcup_{j=1}^{n+1} X_{j}$ with the same sup norm [8]. Put

$$
K=\left\{x \in X_{k}:\left|f_{0}(x)\right|=\left\|f_{0}\right\|_{k}\right\} .
$$

$K$ is a closed set and does not contain $x_{k}$, nor any $x_{n}$ for $n>k$; since $\left(x_{n}\right)$ is an increasing sequence neither does it contain any $x_{n}$ with $n<k$. Let $g$ be a continuous function on $X$ with norm one and such that $g=0$ on $K$ and $g=1$ on $\left\{x_{n}: n \in \mathrm{N}\right\}$. Put $f=g \tilde{f}$. The function $f$ belongs to $\Omega$; however $\left\|f+f_{0}\right\|<\|f\|+\left\|f_{0}\right\|$ since $\left\|f+f_{0}\right\|_{k}<\|f\|_{k}+\left\|f_{0}\right\|_{k}$, so $\Omega_{0}$ is not a peaking set.

In the second case using the same method, and the same function $\tilde{f}$, we construct a continuous function $f$ on $X$ such that

$$
\begin{aligned}
& f(x)=0 \quad \text { for any } x \in X_{k} \text { with }\left|f_{0}(x)+\tilde{f}(x)\right|=\left\|f_{0}\right\|_{k}+\|\tilde{f}\|_{k}, \text { and } \\
& \|f\|_{n}=\theta_{n} f\left(x_{n}\right)=\theta_{n} y_{n}, \quad \text { for any } n \in \mathbb{N} .
\end{aligned}
$$

Again $\left\|f+f_{0}\right\|<\|f\|+\left\|f_{0}\right\|$ since $\left\|f+f_{0}\right\|_{k}<\|f\|_{k}+\left\|f_{0}\right\|_{k}$, so $\Omega_{0}$ is not a peaking set.

Assume now that $\Omega$ is a maximal peaking set. For any $f \in \widetilde{C}(X)$ put

$$
M_{f}=\left\{\left(x_{n}, \theta_{n}\right) \in \prod_{n=1}^{\infty} X_{n} \times \mathbb{K}_{1}:\|f\|_{n}=\theta_{n} f\left(x_{n}\right)\right\}
$$

The sets $M_{f}$ are compact and, since $\Omega$ is a peaking set the family $\left\{M_{f}: f \in \Omega\right\}$ has the finite intersection property, consequently

$$
M=\left\{\left(x_{n}, \theta_{n}\right) \in \prod_{n=1}^{\infty} X_{n} \times \mathbb{K}_{1}:\|f\|_{n}=\theta_{n} f\left(x_{n}\right) \text { for all } f \in \Omega\right\}
$$

is not empty. Assume $M$ contains two distinct points $\left(x_{n}^{1}, \theta_{n}^{1}\right)$ and $\left(x_{n}^{2}, \theta_{n}^{2}\right)$. The sets $\left\{x_{n}^{i}: n \in \mathbb{N}\right\}, i=1,2$ can not have a limit point so we may select $f \in \widetilde{C}(X)$ such that $\left(x_{n}^{1}, \theta_{n}^{1}\right) \in M_{f}$ and $\left(x_{n}^{2}, \theta_{n}^{2}\right) \notin M_{f}$. Then $\Omega \cup\{f\}$ is a peaking set contrary to our assumption that $\Omega$ is maximal. Hence $M$ is a singleton, $M=\left\{\left(x_{n}, \theta_{n}\right)\right\}$, consequently $\left(x_{n}\right)$ is increasing and $\Omega$ is contained in

$$
\Omega\left(\left(x_{n}\right),\left(\theta_{n}\right)\right)=\left\{f \in \tilde{C}(X): \forall n \in \mathbb{N},\|f\|_{n}=\theta_{n} f\left(x_{n}\right)\right\} .
$$

Again from the maximality of $\Omega$, since by the first part of the proof $\Omega\left(\left(x_{n}\right),\left(\theta_{n}\right)\right)$ is a peaking set, we have $\Omega=\Omega\left(\left(x_{n}\right),\left(\theta_{n}\right)\right)$.

LEMMA 3. For any maximal peaking set $\Omega\left(\left(x_{n}\right),\left(\theta_{n}\right)\right)$ we have

$$
\bigcap_{n=1}^{\infty} \operatorname{ker} \delta_{x_{n}} \subset \Omega^{\perp}\left(\left(x_{n}\right),\left(\theta_{n}\right)\right)
$$

where the functional $\delta_{x}$ on $\widetilde{C}(X)$ is defined by $\delta_{x}(h):=h(x)$ for $h \in \widetilde{C}(X)$. 
Proof: Let $g \in \bigcap_{n=1}^{\infty} \operatorname{ker} \delta_{x_{n}}$. For any $n \in \mathbb{N}$ let $U_{n}$ be an open neighbourhood of $x_{n}$ and $k_{n}$ a continuous function on $X$ such that for all $n, m \in \mathbb{N}$

$$
U_{n} \cap U_{m}=\emptyset \text { if } x_{n} \neq x_{m} \text {, and } 0 \leqslant k_{n}(x) \leqslant 1, k_{n}\left(x_{n}\right)=1 \text {, and } k_{n} \equiv 0 \text { on } X \backslash U_{n} \text {. }
$$

Put

$$
f(x):=\sum_{n=1}^{\infty} k_{n}(x) \overline{\theta_{n}}\left(\|g\|_{n}-|g(x)|\right), \text { for } x \in X .
$$

It is clear that $f \in \Omega\left(\left(x_{n}\right),\left(\theta_{n}\right)\right)$. For each $n \in \mathbb{N}$ and $x \in X_{n}$ we have

$$
k_{n}(x)\left(\|g\|_{n}-|g(x)|\right) \leqslant\|g\|_{n}-|g(x)|
$$

so $k_{n}(x)\left(\|g\|_{n}-|g(x)|\right)+|g(x)| \leqslant\|g\|_{n}$, hence

$$
|f(x)|+|g(x)| \leqslant\left|f\left(x_{n}\right)\right|=\|f\|_{n},
$$

which proves that $g \in \Omega^{\perp}\left(\left(x_{n}\right),\left(\theta_{n}\right)\right)$.

Lemma 4. Assume $\mathbb{K}=\mathbb{C}$. Let $f, g$ be elements of $\widetilde{C}(X)$ such that

$$
\exists r>0 \quad \forall \alpha \in \mathbb{K}_{1} \quad\|f+r \alpha g\|=\|f\|
$$

and let $\left(x_{n}\right)$ be an increasing sequence in $X$ such that $\|f\|_{n}=\left|f\left(x_{n}\right)\right|$ for $n \in \mathbb{N}$. Then

$$
g\left(x_{n}\right)=0 \text { for } n \in \mathbb{N} .
$$

Proof: We have

$$
\left\|f+e^{2 \pi i \theta} g\right\|=\sum_{n=1}^{\infty} a_{n}\left\|f+e^{2 \pi i \theta} g\right\|_{n} \geqslant \sum_{n=1}^{\infty} a_{n}\left|f\left(x_{n}\right)+e^{2 \pi i \theta} g\left(x_{n}\right)\right| \stackrel{d f}{=} \xi(\theta) .
$$

By a simple computation one can check that for any nonzero scalar $y$ we have

$$
\int_{0}^{1}\left|1+e^{2 \pi i \theta} y\right| d \theta>1
$$

It follows that if the $g\left(x_{n}\right)$ are not all equal to zero then

$$
\int_{0}^{1} \xi(\alpha) d \alpha=\sum_{n=1}^{\infty} a_{n} \int_{0}^{1}\left|f\left(x_{n}\right)+e^{2 \pi i \alpha} g\left(x_{n}\right)\right| d \alpha>\sum_{n=1}^{\infty} a_{n}\left|f\left(x_{n}\right)\right|=\|f\| .
$$

Hence $\xi\left(\theta_{0}\right)>\|f\|$ for some real number $\theta_{0}$. From (3) $\left\|f+e^{2 \pi i \theta_{0}} g\right\|>\|f\|$ which contradicts our assumption.

From the last two lemmas it follows that in the complex case

$$
\Omega\left(\left(x_{n}\right),\left(\theta_{n}\right)\right)=\bigcap_{n=1}^{\infty} \operatorname{ker} \delta_{x_{n}} .
$$

The above formula is not true in the real case in general. However, the next lemma shows that it remains true if $\left(x_{n}\right)$ is constant. 
Lemma 5. Assume $\Omega=\Omega\left(\left(x_{n}\right),\left(\theta_{n}\right)\right)$ is a maximal peaking set, and assume that the sequence $\left(x_{n}\right)$ contains only one value, that is $x_{n}=x_{1}$ for every $n \in \mathbb{N}$. Then

$$
\Omega^{\perp}\left(\left(x_{n}\right),\left(\theta_{n}\right)\right)=\operatorname{ker} \delta_{x_{1}}
$$

Proof: Suppose that $g \in \widetilde{C}(X)$ satisfies $g\left(x_{1}\right) \neq 0$. Then given any $f \in$ $\Omega\left(\left(x_{n}\right),\left(\theta_{n}\right)\right)$ we can find an arbitrarily small $\alpha \in \mathbb{K}$ such that

$$
\left|f\left(x_{1}\right)\right|<\left|\alpha g\left(x_{1}\right)+f\left(x_{1}\right)\right|
$$

Hence

$$
\|f\|=\sum_{n=1}^{\infty} a_{n}\|f\|_{n}<\sum_{n=1}^{\infty} a_{n}\|\alpha g+f\|_{n}=\|\alpha g+f\|,
$$

so $g \notin \Omega^{\perp}\left(\left(x_{n}\right),\left(\theta_{n}\right)\right)$. This implies that $\Omega^{\perp}\left(\left(x_{n}\right),\left(\theta_{n}\right)\right) \subset \operatorname{ker} \delta_{x_{1}}$. The other inclusion follows from Lemma 3 .

LEMMA 6. Assume that $\mathbb{K}=\mathbb{R}$ and that $\Omega=\Omega\left(\left(x_{n}\right),\left(\theta_{n}\right)\right)$ is a maximal peaking set. Then

$$
\Omega^{\perp}\left(\left(x_{n}\right),\left(\theta_{n}\right)\right)=\operatorname{ker}\left(\sum_{n=1}^{\infty} a_{n} \theta_{n} \delta_{x_{n}}\right)
$$

PROOF: If the sequence $\left(x_{n}\right)$ is constant the lemma follows from the previous one so we may assume that $\left(x_{n}\right)$ is not constant. For each $n \in \mathbb{N}$, let $U_{n}$ be an open neighbourhood of $x_{n}$ such that $U_{n} \cap U_{m}=\emptyset$ if $x_{n} \neq x_{m}$. Let $g \in \widetilde{C}(X)$ be such that

$$
\|g\|_{n}=\left|g\left(x_{n}\right)\right| \text { for } n \in \mathbb{N}, \sum_{n=1}^{\infty} a_{n} \theta_{n} g\left(x_{n}\right)=0, \text { and } g \equiv 0 \text { on } X \backslash\left(\bigcup_{n=1}^{\infty} U_{n}\right) \text {. }
$$

If $\left(x_{n}\right)$ contains at least two values then we can always find a $g$ that is not equal to zero at these two points. Moreover, if $h \in \tilde{C}(X)$ is such that $\sum_{n=1}^{\infty} a_{n} \theta_{n} h\left(x_{n}\right)=0$, then we can always find a function $g$ that satisfies all the above conditions and a function $g^{\prime} \in \bigcap_{n=1}^{\infty} \operatorname{ker} \delta_{x_{n}}$ such that $g+g^{\prime}=h$. From Lemma 3 we know that $\Omega^{\perp}\left(\left(x_{n}\right),\left(\theta_{n}\right)\right)$ contains $\bigcap_{n=1}^{\infty} \operatorname{ker} \delta_{x_{n}}$ so to prove the lemma it is sufficient to show that the function $g$ is contained in $\Omega^{\perp}\left(\left(x_{n}\right),\left(\theta_{n}\right)\right)$.

Let $f \in \Omega\left(\left(x_{n}\right),\left(\theta_{n}\right)\right)$ be such that for some sequence of numbers $\left(c_{n}\right)$ with absolute values equal to one, we have

$$
\begin{aligned}
& f(x)=c_{n} g(x) \quad \text { if } x \in U_{n} \text { and } g\left(x_{n}\right) \neq 0, \\
& f(x)=0 \quad \text { if } x \in X \backslash\left(\bigcup_{n=1}^{\infty} U_{n}\right) .
\end{aligned}
$$


Notice that $c_{n}$ is positive if and only if $g\left(x_{n}\right) \theta_{n}$ is positive. For any $0 \leqslant r \leqslant 1$ we have

$$
\begin{aligned}
& \|f \pm r g\|_{n}=\left(1 \pm \frac{r}{c_{n}}\right)\left|f\left(x_{n}\right)\right| \text { if } g\left(x_{n}\right) \neq 0, \text { and } \\
& \|f \pm r g\|_{n}=\|f\|_{n} \text { otherwise, }
\end{aligned}
$$

hence

$$
\begin{aligned}
\|f \pm r g\| & =\sum_{n=1}^{\infty} a_{n}\|f \pm r g\|_{n}=\sum_{n=1}^{\infty} a_{n}\|f\|_{n} \pm r \sum_{n=1}^{\infty} a_{n} \operatorname{sgn}\left(c_{n}\right)\left|g\left(x_{n}\right)\right| \\
& =\|f\| \pm r \sum_{n=1}^{\infty} a_{n} \theta_{n} g\left(x_{n}\right)=\|f\|,
\end{aligned}
$$

so $g \in \Omega^{\perp}\left(\left(x_{n}\right),\left(\theta_{n}\right)\right)$.

Proof of THEOREM 1: Notice that the isometry $T$ maps maximal peaking sets onto maximal peaking sets. We shall show that $T$ maps the maximal peaking sets $\Omega\left(\left(x_{n}\right),\left(\theta_{n}\right)\right)$ that correspond to the constant sequence $\left(x_{n}\right)=\left(x_{1}\right)$, onto the same type of maximal peaking sets: $T\left(\Omega\left(\left(x_{1}\right),\left(\theta_{1}\right)\right)\right)=\Omega\left(\left(x_{1}^{\prime}\right),\left(\theta_{1}^{\prime}\right)\right)$. We shall also show that $x_{1}^{\prime}$ does not depend on the value of $\theta_{1}$, but only on $x_{1}$.

In the complex case, by the previous lemmas, $\Omega^{\perp}\left(\left(x_{n}\right),\left(\theta_{n}\right)\right)$ is of codimension one if and only if $\left(x_{n}\right)$ is constant. Since $T$ preserves the codimension and the maximal peaking sets and their complements, it maps maximal peaking sets that correspond to constant sequences in $X$, onto the same type maximal peaking sets. For a constant sequence $\left(x_{n}\right)$, $\Omega^{\perp}\left(\left(x_{n}\right),\left(\theta_{n}\right)\right)$ is equal to $\operatorname{ker} \delta_{x_{1}}$ and hence it does not depend on $\theta_{1}$.

In the real case the situation is more complicated - by the previous lemma

$$
\Omega^{\perp}\left(\left(x_{n}\right),\left(\theta_{n}\right)\right)=\operatorname{ker}\left(\sum_{n=1}^{\infty} a_{n} \theta_{n} \delta_{x_{n}}\right)
$$

for any increasing sequence $\left(x_{n}\right)$, so the codimension of $\Omega^{\perp}\left(\left(x_{n}\right),\left(\theta_{n}\right)\right)$ is always one. Since $T$ preserves the maximal peaking sets and their complements as well as the norm, from (4) we get that for any $\left(\left(x_{n}\right),\left(\theta_{n}\right)\right)$ there is a $\left(\left(x_{n}^{\prime}\right),\left(\theta_{n}^{\prime}\right)\right)$ in $X^{\prime} \times K_{1}$ such that

$$
T^{*}\left(\sum_{n=1}^{\infty} a_{n} \theta_{n} \delta_{x_{n}}\right)=c \sum_{n=1}^{\infty} a_{n}^{\prime} \theta_{n}^{\prime} \delta_{x_{n}^{\prime}}
$$

where $c=\left(\sum_{n=1}^{\infty} a_{n}\right) /\left(\sum_{n=1}^{\infty} a_{n}^{\prime}\right)$, and $T^{*}$ is the conjugate of $T$. Denote by $\Xi$ the set of all functionals of the form $\sum_{n=1}^{\infty} a_{n} \theta_{n} \delta_{x_{n}}$. We shall say that functionals $F_{1}, F_{2} \in \Xi$ are orthogonal if

$$
\left\|F_{1}\right\|+\left\|F_{2}\right\|=\left\|F_{1}+F_{2}\right\|=\left\|F_{1}-F_{2}\right\|
$$

For $F \in \Xi$ we put

$$
F^{\perp}=\left\{F_{1} \in \Xi: F_{1} \text { and } F \text { are orthogonal }\right\}
$$


Since the definition of orthogonality involves only the norm and the linear structure it is preserved by $T^{*}$. On the other hand $F_{1}=\sum_{n=1}^{\infty} a_{n} \theta_{n} \delta_{x_{n}}$ and $F_{2}=\sum_{n=1}^{\infty} a_{n} \tilde{\theta}_{n} \delta_{\widetilde{x}_{n}}$ are orthogonal if and only if the corresponding sequences $\left(x_{n}\right)$ and $\left(\tilde{x}_{n}\right)$ do not have any common point. Put

$$
\Xi_{0}=\left\{F^{\perp}: F \in \Xi\right\} \text {. }
$$

The set $\Xi_{0}$ is partially ordered by inclusion, $F^{\perp}$ is a maximal element of $\Xi_{0}$ if and only if $F$ is defined by a constant sequence $\left(x_{n}\right)=\left(x_{1}\right)$. All this is again preserved by $T$, so $T$ maps $\operatorname{ker} \delta_{x}, x \in X_{1}$, onto ker $\delta_{x^{\prime}}, x^{\prime} \in X_{1}^{\prime}$.

We proved that in both the complex and the real case there is a bijection $\varphi_{1}$ from $X_{1}^{\prime}$ onto $X_{1}$ such that $T\left(\operatorname{ker} \delta_{\varphi_{1}(x)}\right)=\operatorname{ker} \delta_{x}$, for $x \in X_{1}^{\prime}$, equivalently $T^{*}\left(\delta_{x}\right)=\kappa_{1}(x) \delta_{\varphi_{1}(x)}$, for $x \in X_{1}^{\prime}$, or

$$
T(f)(x)=\kappa_{1}(x) f\left(\varphi_{1}(x)\right), \text { for } x \in X_{1}^{\prime}
$$

where $\kappa_{1}$ has the constant absolute value equal to $c=\left(\sum_{n=1}^{\infty} a_{n}\right) /\left(\sum_{n=1}^{\infty} a_{n}^{\prime}\right)$. Since $T^{*}$ is continuous in the weak* topology the functions $\varphi_{1}$ and $\kappa_{1}$ must also be continuous.

We proved that $T$ has the canonical form on $X_{1} \subset X$, and

$$
\|T f\|_{1}=c\|f\|_{1} \text { fór } f \in \widetilde{C}(X)
$$

Now we need to extend $\varphi_{1}$ and $\kappa_{1}$ to the entire set $X$. Because of the symmetry we may assume that

$$
\frac{a_{1}}{a_{1}^{\prime}} \leqslant \frac{\sum_{n=1}^{\infty} a_{n}}{\sum_{n=1}^{\infty} a_{n}^{\prime}}
$$

and define new norms on $\widetilde{C}(X)$ and $\widetilde{C}\left(X^{\prime}\right)$, by

$$
\begin{aligned}
& p(f)=\|f\|-a_{1}\|f\|_{1}=\sum_{n=2}^{\infty} a_{n}\|f\|_{n}, \text { for } f \in \widetilde{C}(X), \text { and } \\
& p^{\prime}(g)=\|g\|-\frac{a_{1}}{c}\|g\|_{1}=\left(a_{1}^{\prime}-\frac{a_{1}}{c}\right)\|g\|_{1}+\sum_{n=2}^{\infty} a_{n}^{\prime}\|g\|_{n}, \text { for } g \in \widetilde{C}\left(X^{\prime}\right) .
\end{aligned}
$$

By (6) $T$ is an isometry of $(\widetilde{C}(X), p(\cdot))$ onto $\left(\widetilde{C}\left(X^{\prime}\right), p^{\prime}(\cdot)\right)$. If $a_{1}^{\prime}-\left(a_{1} / c\right) \neq 0$, then, based on what we already proved applied to this new isometry, there is a homeomorphism $\varphi_{2}$ from $X_{1}^{\prime}$ onto $X_{2}$, and a scalar valued function $\kappa_{2}$ such that

$$
T(f)(x)=\kappa_{2}(x) f\left(\varphi_{2}(x)\right), \text { for } x \in X_{1}^{\prime},
$$

but this contradicts (5). Hence $a_{1}^{\prime}-\left(a_{1} / c\right)=0$ and $\varphi_{2}$ and $\kappa_{2}$, are extensions to $X_{2}^{\prime}$ of $\varphi_{1}$ and $\kappa_{1}$, respectively. By continuing this process we extend $\varphi_{1}$ to a homeomorphism of $X^{\prime}$ onto $X$. 
We also get

$$
\frac{a_{1}}{a_{1}^{\prime}}=\frac{\sum_{n=1}^{\infty} a_{n}}{\sum_{n=1}^{\infty} a_{n}^{\prime}}, \quad \frac{a_{2}}{a_{2}^{\prime}}=\frac{\sum_{n=2}^{\infty} a_{n}}{\sum_{n=2}^{\infty} a_{n}^{\prime}}, \ldots,
$$

hence for $c=\left(\sum_{n=1}^{\infty} a_{n}\right) /\left(\sum_{n=1}^{\infty} a_{n}^{\prime}\right)$ we have

$$
a_{n}=c a_{n}^{\prime}, \text { for } n \in \mathbb{N}
$$

THEOREM 7. Let $(C(X), d(\cdot, \cdot))$ be the metric vector space of all continuous functions on a $\sigma$-compact set $X$, where the metric is given by (2). Let $\left(C\left(X^{\prime}\right), d(\cdot, \cdot)\right)$, be the analogous space on $X^{\prime}$. Assume that $T$ is a linear isometry from $C(X)$ onto $C\left(X^{\prime}\right)$. Then there is a homeomorphism $\varphi$ of $X^{\prime}$ onto $X$ with $\varphi\left(X_{n}^{\prime}\right)=X_{n}$, for $n \in \mathrm{N}$, and a unimodular, continuous, scalar valued function $\kappa$ on $X^{\prime}$ such that

$$
T f=c \kappa f \circ \varphi \text { for all } f \in C(X),
$$

where $c=\left(\sum_{n=1}^{\infty} a_{n}\right) /\left(\sum_{n=1}^{\infty} a_{n}^{\prime}\right)$. Moreover $a_{n}=c a_{n}^{\prime}$, for $n \in \mathbb{N}$.

Proof: Let $T: C(X) \rightarrow C\left(X^{\prime}\right)$ be a surjective isometry. For a fixed $f \in C(X)$ we define a function $\alpha_{f}: \mathbb{R}^{+} \rightarrow \mathbb{R}^{+}$by

$$
\alpha_{f}(t) \stackrel{d f}{=} d(t f, 0)=\sum_{n=1}^{\infty} a_{n} \frac{\|t f\|_{n}}{1+\|t f\|_{n}}=\sum_{n=1}^{\infty} a_{n} \frac{t\|f\|_{n}}{1+t\|f\|_{n}} .
$$

It is easy to check that

$$
\frac{d \alpha_{f}}{d t}=\sum_{n=1}^{\infty} a_{n} \frac{\|f\|_{n}}{\left(1+\|t f\|_{n}\right)^{2}}, \text { for } t \geqslant 0
$$

For $t=0$ the value of $\frac{d \alpha_{f}}{d t}$ is $\sum_{n=1}^{\infty} a_{n}\|f\|_{n}$; it may by finite or may be equal to $+\infty$. Since the function $\alpha_{f}$ is defined only in terms of the metric and linear structure it is preserved by the isometry. Hence $\alpha_{f}=\alpha_{T j}$ and $T$ preserves the quantity

$$
\frac{d \alpha_{f}}{d t}(0)=\|f\|=\sum_{n=1}^{\infty} a_{n}\|f\|_{n} .
$$

Consequently $T$ restricted to the Banach space

$$
\widetilde{C}(X)=\left\{f \in C(X):\|f\|=\sum_{n=1}^{\infty} a_{n}\|f\|_{n}<\infty\right\}
$$

is an isometry, with respect to that Banach space norm, onto

$$
\widetilde{C}\left(X^{\prime}\right)=\left\{g \in C\left(X^{\prime}\right):\|g\|=\sum_{n=1}^{\infty} a_{n}^{\prime}\|g\|_{n}<\infty\right\} .
$$


By the previous Theorem there is a homeomorphism $\varphi$ of $X^{\prime}$ onto $X$ with $\varphi\left(X_{n}^{\prime}\right)=X_{n}$, for $n \in \mathrm{N}$, and a unimodular, continuous, scalar valued function $\kappa$ on $X^{\prime}$ such that

$$
T f=\kappa f \circ \varphi \text { for all } f \in \widetilde{C}(X) \text {. }
$$

Since $\tilde{C}(X)$ is dense in $(C(X), d(\cdot, \cdot))$ the above formula holds for all functions from $C(X)$.

\section{REFERENCES}

[1] E. Behrends, $M$-structure and the Banach-Stone theorem, Lecture Notes in Mathematics 736 (Springer-Verlag, Berlin, Heidelberg, New York, 1979).

[2] E. Behrends and U. Schmidt-Bichler, ' $M$-structure and the Banach-Stone theorem', Studia Math. 69 (1980), 33-40.

[3] M. Cambern, 'A Banach-Stone theorem for spaces of weak* continuous functions', Proc. Royal Soc. Edinburgh 101 (1985), 203-206.

[4] M. Cambern and K. Jarosz, 'The isometries of $H_{H}^{1}$ ', Proc. Amer. Math. Soc. 107 (1989), 205-214.

[5] M. Cambern and K. Jarosz, 'Isometries of spaces of weak * continuous functions', Proc. Amer. Math. Soc. 106 (1989), 707-712.

[6] M. Cambern and K. Jarosz, 'Multipliers and isometries in $H_{E}^{\infty}$ ', Bull. London Math. Soc. 22 (1990), 463-466.

[7] M. Cambern and V.D. Pathak, 'Isometries of spaces of differentiable functions', Math. Japon. 26 (1981), 253-260.

[8] R. Engelking, General Topology, Monografie Matematyczne 60 (Polish Scientific Publishers, Warsaw, 1977).

[9] K. Jarosz, 'Any Banach space has an equivalent norm with trivial isometries', Israel $J$. Math. 64 (1988), 49-56.

[10] K. Jarosz and V. Pathak, 'Isometries and small bound isomorphisms of function spaces', in Function spaces, (K. Jarosz, Editor), Lecture Notes in Pure and Applied Maths. 136 (Marcel Dekker, New York, 1991), pp. 241-271.

[11] K.D. Leeuw, W. Rudin and J. Wermer, 'Isometries of some functions spaces', Proc. Amer. Math. Soc. 11 (1960), 694-698.

[12] P.K. Lin, 'The isometries of $H^{\infty}(E)$ ', Pacific J. Math. 143 (1990), 69-77.

[13] N.V. Rao and A.K. Roy, 'Linear isometries of some function spaces', Pacific J. Math. 38 (1971), 177-192.

Faculty of Science

University of Cantabria

39071 Santander

Spain
Department of Mathematics and Statistics

Southern Illinois University

Edwardsville IL 62026-1653

United States of America 